\title{
Primary gastric low-grade malt lymphoma: evaluation of clinical and laboratorial factors for achieving complete remission
}

\begin{abstract}
Introduction: Gastric mucosa associated lymphoid tissue (MALT) lymphoma, is an indolent cancer that occurs in the presence of pre-existing prolonged lymphoid proliferation in mucosal sites. In that instance, the most well established trigger is the gastric Helicobacter pylori $(\mathrm{Hp})$ infection.
\end{abstract}

Aim: Evaluation of clinical and laboratorial factors and the effect of therapy in achieving Complete Remission (CR) in patients with gastric MALT lymphoma.

Methods: Multi centric study from a geographic area (Southern Portugal), in which clinical and pathological features of patients with gastric MALT lymphoma, according to Dawson criteria for primary gastrointestinal lymphoma, were evaluated retrospectively with a follow-up $\geq 12$ months. Lugano system was used for staging. Statistical analysis was performed using Kaplan-Meyer curves and bivariate analysis with Mann-Whitney test. A p-value of $\leq 0.05$ was considered statistically significant.

Results: A total of 34 cases were identified, 23 men, and 11 women, with an average age of 59 years. The most common symptom was epigastric pain $(74 \%) ; 6$ patients presented gastrointestinal bleeding and 3 patients had type B symptoms. The most prevalent localization was gastric body; 18 patients had an ulcerative pattern at endoscopy. At diagnosis, 91\% and 94\% were classified as limited disease (Lugano I-II and Ann Arbor IE-IIE respectively) and $94 \%$ had an International Prognostic Index (IPI) $<3$. A total of 20 patients $(59 \%)$ had histological evidence of $\mathrm{Hp}$ infection. CR rate at one year was $77 \%$ ( $70 \%$ in the Hp-positive group versus $86 \%$ in the Hp-negative group), with an average remission time of 6 months. Using Lugano Staging System, CR was achieved in $77 \%$ of patients with limited disease versus $67 \%$ in those with advanced disease. In the Hp-positive group, isolated antibiotic the rapyled to a CR in $60 \%$ of the cases, including one patient with advanced disease. One patient of the Hp-negative group achieved CR with isolated antibiotic therapy. The variables Limited Disease, IPI score $<3$, age, histological evidence of Hp, serum LDH, albumin and $\beta 2$-microglobulin levels, were not associated with one year rate of CR $(\mathrm{p}>0.05)$.

Conclusion: The results confirm the good prognosis and the indolence of MALT lymphoma. The variables studied couldn't predict CR in 1 year, but enhance the idea that patients with early stage Hp-negative MALT lymphoma and with advanced disease, might still benefit from antibiotic treatment.
Volume 2 Issue 2 - 2015

\author{
Daniel Trabulo,' Artur Antunes, ${ }^{2}$ Suzane \\ Ribeiro,' Marta Eusébio, ${ }^{2}$ Bruno Piexe, ${ }^{2}$ \\ Horácio Guerreiro, ${ }^{2}$ Ana Paula Oliveira' \\ 'Gastroenterology department, Hospital de São Bernardo, \\ Portugal \\ ${ }^{2}$ Gastroenterology department, Hospital de Faro, Portugal
}

Correspondence: Daniel Trabulo, Gastroenterology Department, Hospital de Sao Bernardo, Centro Hospitalar de Setubal, EPE, Rua Camilo Castelo Branco, 2910-446 Setubal, Portugal,Tel 0035I 968I48206,Email danieltrabulo@yahoo.com

Received: January 30, 2015 | Published: April 2, 2015
Abbreviations: MALT, mucosa associated lymphoid tissue; Hp, helicobacter pylori; CR, complete remission; IPI, international prognostic index; CT, computed tomography; EUS, endoscopic ultrasound; PPI, proton pump inhibitor; RT, radiotherapy

\section{Introduction}

The gastrointestinal tract is the predominant site of primary extranodal lymphomas. Primary gastric lymphomas are rare and account for less than $3 \%$ of all gastric cancers and $10 \%$ of lymphomas. ${ }^{1}$ The most common subtype is Gastric Mucosa Associated Lymphoid Tissue (MALT) lymphoma, now called extranodal marginal zone B-cell lymphoma of MALT type in the REAL classification. ${ }^{2}$ It is characterized for being an indolent cancer that occurs in the presence of pre-existing prolonged lymphoid proliferation in mucosal sites. However, transformation to high-grade lymphomas has been described by several authors. ${ }^{2-4}$
Compared with node-based lymphomas, gastric MALT lymphoma is recognized as a distinct entity with a characteristic presentation, histological spectrum and clinical behavior. An overwhelming body of evidence has shown that development of MALT lymphomas is due to the clonal expansion of $\mathrm{B}$ cells that accompanies chronic gastritis in the presence of Helicobacter pylori (Hp). Most patients (>90\%) with gastric MALT lymphoma are Hp positive..$^{3-7}$ The most dramatic evidence supporting a pathogenic role for Hp in MALT lymphoma is remission of the tumor following eradication of $\mathrm{Hp}$ with antibiotic therapy. In fact, anti biotherapy directed at Hp infection results in regression of most early lesions and has become the treatment of choice for most patients. ${ }^{6-8}$ The aim of this study is to evaluate clinical, epidemiological, endoscopic and laboratorial characteristics of gastric MALT lymphomas and to determine clinical and laboratorial factors, as well as the effect of therapy in achieving Complete Remission (CR) in a Portuguese population. 


\section{Patients and methods}

A multi centric study was conducted from a geographic region (southern Portugal), which included two centers: Hospital de Sao Bernardo, Centro Hospitalar de Setubal and Hospital de Faro, Centro Hospitalar do Algarve. During the period from 2005 to 2012, clinical files of patients with the diagnosis of primary gastric MALT lymphoma from these institutions were retrospectively analyzed. The diagnosis of Primary Gastrointestinal Lymphoma was defined by Dawson criteria ${ }^{9}$ and CR was defined using GELA scoring system (Groupe d' Etude des Lymphomes de 1' Adulte). ${ }^{10}$ Only "naïve patients" were included. Evaluation for Hp infection was made by histology (8-10 tissue biopsies from both the antrum and body). Staging was made according to Lugano and Ann Arbor staging systems. International Prognostic Index (IPI) was used for risk assessment. Lugano staging system was used to determine the treatment. The following data were obtained: age, gender, symptoms, complete blood count, biochemical studies (lactate dehydrogenase, $\beta 2$-microglobulin, albumin, and uric acid), topography, endoscopic appearance, Hp infection and treatment modalities. Contrast-enhanced computed tomography (CT) scan of the chest, abdomen and pelvis and endoscopic ultrasound (EUS) were performed to evaluate distant disease and involvement of gastric lymph nodes. The period of follow-up was $\geq 12$ months. Statistical analysis was performed using Kaplan-Meyer curves and bivariate analysis with Mann-Whitney test. A p-value of $\leq 0.05$ was considered statistically significant.

\section{Results}

A total of 34 cases were identified, 23 men and 11 women, with an average age of $59 \pm 14$ years. The most common symptom was epigastric pain $(74 \%)$, followed by nausea/vomiting $(69 \%)$ and anorexia (50\%). Six patients (18\%) presented gastrointestinal bleeding and three patients ( $9 \%$ ) had type B symptoms (Figure 1). The duration of symptoms preceding the diagnosis was variable, ranging from 3 days to 4 years. The physical examination was often normal, but it revealed abdominal distension in $6 \%$ and signs of gastric perforation in $3 \%$ of the cases. The most prevalent localization was gastric body $(60 \%)$, followed by antrum $(30 \%)$. At endoscopic presentation, 18 patients $(53 \%)$ had an ulcerative pattern; $35 \%$ had an infiltrative pattern and $12 \%$ had a polyploidy appearance (Figure 2).

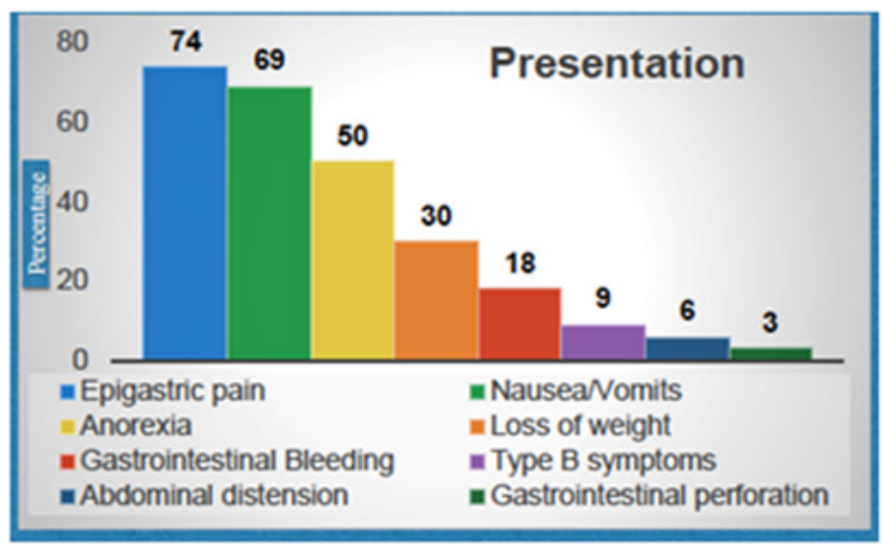

Figure I Clinical Presentation of Primary Gastric MALT lymphomas.

Laboratory studies were often normal at presentation. The main results were elevated LDH (14 patients), elevated $\beta 2$-microglobulin (12 patients) and hypoalbuminemia ( 8 patients). Anemia was present in 5 patients (Figure 3). A total of 20 patients (59\%) had histological evidence of Hp infection. At diagnosis, 91 and 94\% were classified as early disease (Lugano I-II and Ann Arbor IE-IIE, respectively); and $94 \%$ had an IPI $<3$ (Figure 4). All patients were treated with Hp eradication therapy at diagnosis, independently of Hp infection. Thirteen patients $(38 \%)$ were only treated with antibiotics combined with proton pump inhibitor (PPI). The most common regimen used was amoxicillin 1g (two times daily), clarithromycin $500 \mathrm{mg}$ (two times daily) and standard dose of PPI (two times daily). Eight patients $(24 \%)$ were treated with eradication therapy followed by local radiation therapy and 5 patients $(15 \%)$ were treated with eradication therapy followed by chemotherapy (cyclophosphamide or Chlorambucil). Immunotherapy (Rituximab) was used in 3 patients $(9 \%)$. Surgery was necessary in 2 patients $(6 \%)$, due to perforation and bleeding that could not be controlled by endoscopy, before the establishment of the diagnosis of lymphoma (Figure 5).

\section{Endoscopic Pattern}

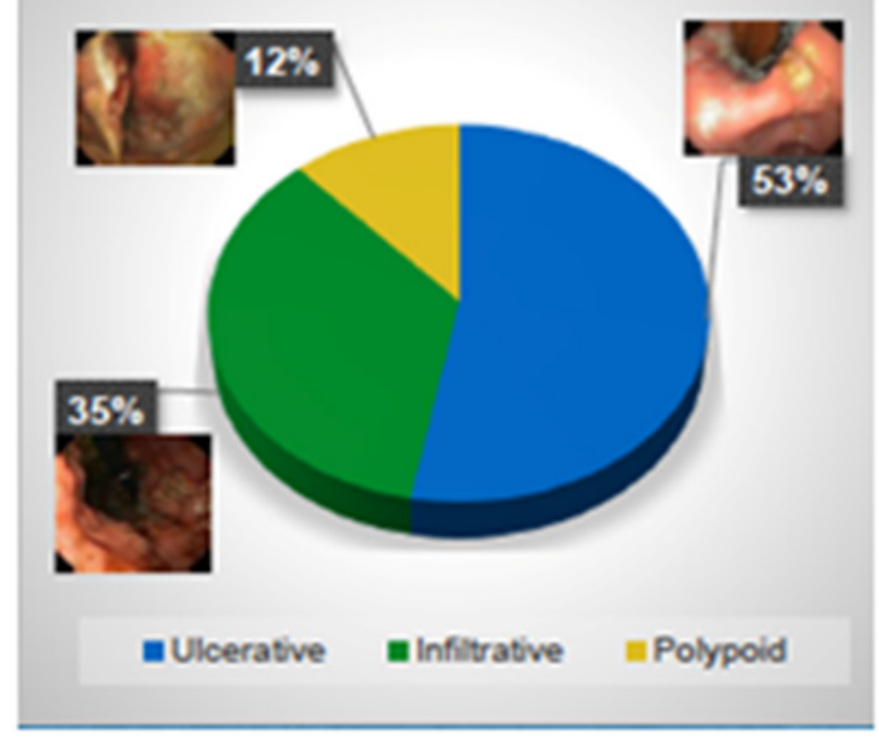

Figure 2 Endoscopic Pattern of Primary Gastric MALT lymphomas.

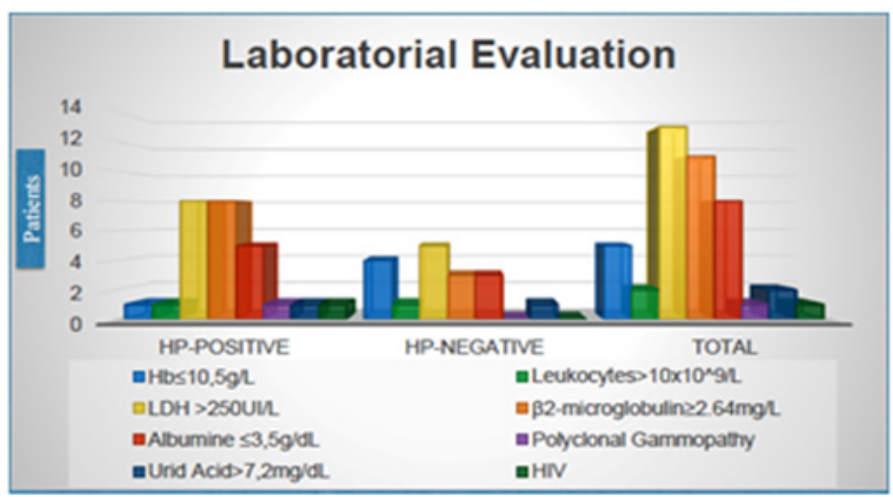

Figure 3 Laboratorial evaluation of Primary Gastric MALT lymphomas.

Seventy-seven percent of all patients achieved CR at one year, with an average remission time of 6 months. In the second year of follow-up, only 4 patients (12\%) failed CR (average remission time of 8,5 months). CR at one year was achieved in $70 \%$ in the Hp-positive 
group (67\% in the Hp-positive limited-disease) versus $86 \%$ in the Hp-negative group (92\% in the Hp-negative limited-disease). CR was achieved in $77 \%$ of patients with limited disease (Lugano I-II) versus $67 \%$ in those with advanced disease (Lugano IV) (Figure 6). In the $\mathrm{Hp}$-positive group, eradication therapy led to $\mathrm{CR}$ in $60 \%$ of cases, including in one patient with advanced disease. Of notice, CR was also achieved in one Hp-negative patient, treated only with antibiotics and proton-pump inhibitors (PPIs).

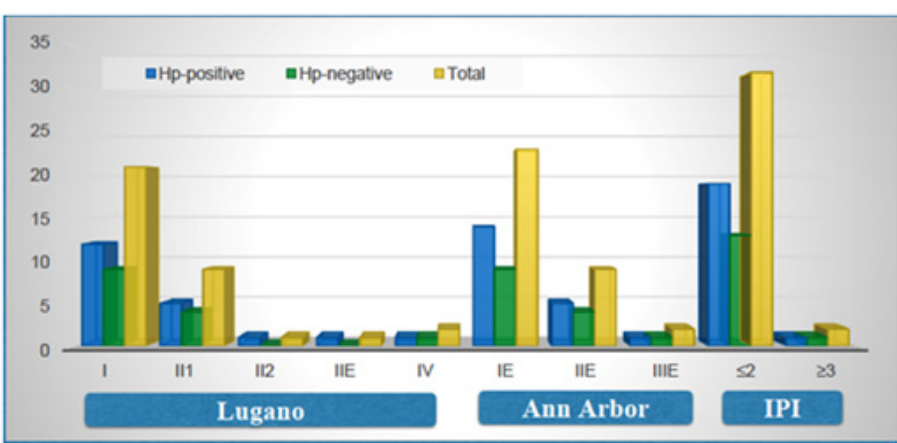

Figure 4 Staging and risk assessment.

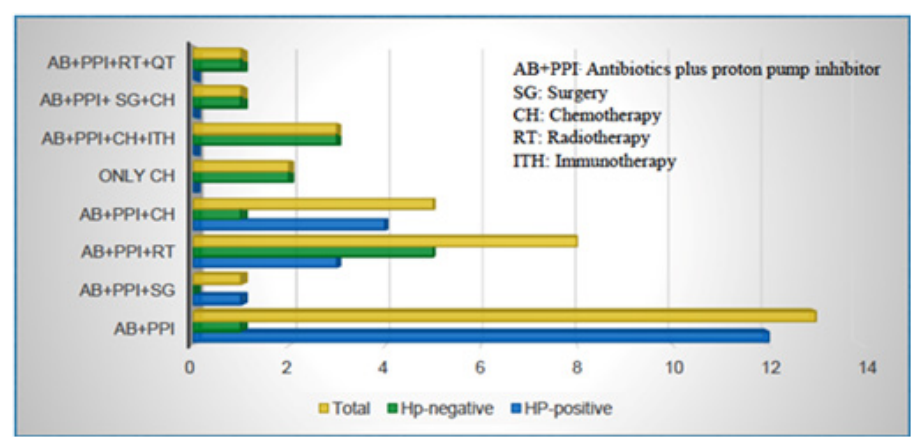

Figure 5 Treatment modalities.
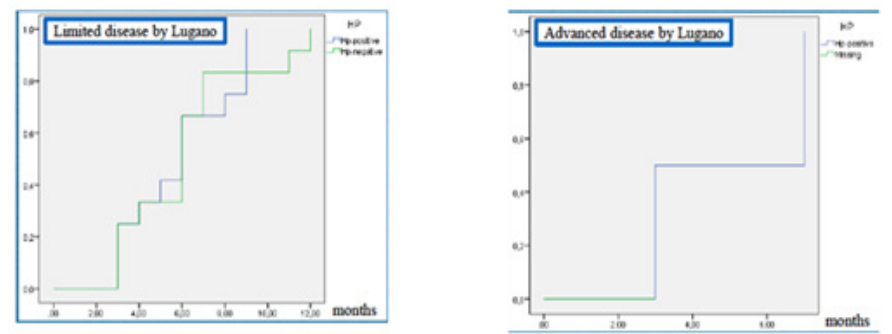

Figure 6 Remission according to Lugano Staging.

At one year follow-up, although reaching remission, there was one death in an Hp positive patient, without direct relation with MALT lymphoma. In our study, we tried to evaluate if there were clinical and laboratorial factors that could predict $\mathrm{CR}$ in one-year. These factors were: age $\leq 60$ years; leukocytes $\leq 10 \times 10^{9} / \mathrm{L}$; albumin $\geq 3.5 \mathrm{~g} / \mathrm{dL} ; \beta 2$ microglobulin $<2.64 \mathrm{mg} / \mathrm{L} ; \mathrm{LDH} \leq 250 \mathrm{UI} / \mathrm{L}, \mathrm{Hp}$ positive; IPI score $<3$; limited disease (Lugano I-II). A trend for CR at one year was observed for the variables: age $\geq 60$ years, low leukocyte count, IPI score $<3$ and limited disease, but it didn't reach statistical significance after bivariate analysis $(\mathrm{p}>0,05)$ (Table 1$)$.

\section{Discussion}

The stomach is the most common extranodal site of lymphoma and accounts for $68-75 \%$ of GI lymphomas. The majority of gastric lymphomas are generally equally divided in two subtypes: extranodal marginal zone B-cell lymphoma of mucosa-associated lymphoid tissue (MALT) type (previously called low-grade MALToma, MALT-type lymphoma, or MALT lymphoma) in 38-48\%; and diffuse B-cell lymphoma in $45-59 \%$ of the cases. ${ }^{2-9,11}$ Gastric MALT lymphoma reaches its peak incidence between the ages of 55-60 years with a slight male predominance. ${ }^{12}$ Patients typically present with nonspecific symptoms, frequently seen in more common gastric diseases, such as peptic ulcer disease, Adenocarcinoma and non-ulcer dyspepsia. The most common presenting symptoms include epigastric pain $(78-93 \%)$, anorexia (47\%), weight loss $(25 \%)$, nausea/vomiting $(18 \%)$ and occult gastrointestinal bleeding (19\%). Systemic B symptoms (fever and night sweats) are seen in $12 \%$. Weight loss is not always considered a B symptom because is due frequently to gastric involvement. The duration of symptoms preceding the diagnosis can range from a few days to six years. ${ }^{11-15}$ Physical examination is often normal, but may reveal peripheral lymph Adenopathy or a palpable mass when there is advanced disease. ${ }^{11-13}$ Laboratory tests should include a complete blood count, $\beta 2$-microglubulin, LDH, HIV, hepatitis $\mathrm{B}$ and $\mathrm{C}$ serologies, liver and renal function and protein electrophoresis. ${ }^{7}$ Anemia and elevated erythrocyte sedimentation ratio could be present. ${ }^{11-15}$ The diagnosis is made upon histological criteria and presence of B-cell markers by Immuno cytochemistry. ${ }^{16}$

Table I Evaluation of clinical and laboratorial factors for achieving complete remission in one-year

\begin{tabular}{llll}
\hline & Remission & $\begin{array}{l}\text { No } \\
\text { Remission }\end{array}$ & $\begin{array}{l}\text { Bivariate } \\
\text { analysis' }\end{array}$ \\
\hline $\begin{array}{l}\text { Age } \leq 60 \\
\text { Leukocytes } \leq 10 \times\end{array}$ & 16 & 2 & 0,074 \\
$\begin{array}{l}\text { I09/L } \\
\text { Albumine } \geq 3,5 g / d L\end{array}$ & 14 & 7 & 0,07 I \\
$\begin{array}{l}\text { B2-microglobulin } \\
<2.64 \mathrm{mg} / \mathrm{L}\end{array}$ & 13 & 5 & 0,439 \\
$\begin{array}{l}\text { LDH } \leq 250 \mathrm{UI} / \mathrm{L} \\
\text { Hp-positive }\end{array}$ & 9 & 3 & 0,608 \\
$\begin{array}{l}\text { IPI score }<3 \\
\begin{array}{l}\text { Limited Disease } \\
\text { (Lugano) }\end{array}\end{array}$ & 14 & 5 & 0,546 \\
\hline
\end{tabular}

Several studies have demonstrated a strong association between Hp infection and MALT lymphoma, specifically with Hp strains expressing the CagA protein. ${ }^{5}$ In fact, Hp-induced gastritis first leads to accumulation of $\mathrm{CD} 4+$ lymphocytes and mature B cells in lamina propria. Antigens derived from $\mathrm{Hp}$ drive the activation of T cells, B cell proliferation and lymphoid follicle formation, which if persistent can evolve into a monoclonal lymphoma. ${ }^{3,4,17}$ It is also possible that other species of $\mathrm{Hp}$ are involved in the development of gastric MALT lymphomas, such as H. heilmanni. ${ }^{18}$ Patients should be tested for Hp which can be detected by histologic specimen, biopsy urease test, urea breath test, stool antigen test or serology. ${ }^{19}$ The prevalence of Hp infection is variable according to studies (72-98\%). ${ }^{3-7}$ In our study, the prevalence was $59 \%$. The most probable explanation is a missed diagnosis caused by a single test used for the diagnosis of $\mathrm{Hp}$ infection (histology) and its variable distribution in the stomach leading to falsenegative results. Moreover, we didn't have in account the use of PPIs that could also influence the results. Furthermore, there are cases of 
MALT lymphomas in which Hp infection is not demonstrated due to influence of environmental, genetic and alimentary factors).$^{20}$ Therapy directed at the Hp infection results in regression of most early lesions and has become the treatment of choice for most patients $(70-80 \%){ }^{6-8}$

The diagnosis of gastric MALT lymphoma is established during upper endoscopy with multiple biopsies (minimum 10 biopsies for visible lesions). Jumbo biopsies, snare biopsies, biopsies within biopsies and needle aspiration can increase yeld. Both suspicious appearing lesions and normal appearing mucosa should be biopsied since gastric MALT lymphoma can occasionally present as multifocal disease with involvement of tissue that appears to be unaffected. This procedure named gastric mapping is recommended by the German S3 guideline as well as in the European EGILs Consensus Report of 2011.,21 Findings on upper endoscopy are diverse and may include mucosal erythema, mass or polyploidy lesion with or without ulceration, gastric ulcer, nodularity or thickened cerebroid gastric folds. ${ }^{11-15}$ An EUS should determine the depth of invasion and perigastric lymph nodes. Usually, the pattern seen on EUS is superficial spreading or diffuse infiltrating lesions., ${ }^{7,22}$ A contrastenhanced computed tomography (CT) scan of chest, abdomen and pelvis should be performed to evaluate distant disease. Bone marrow biopsy and aspirate are strongly recommended. ${ }^{7}$

The Ann Arbor staging system used for most lymphomas is considered to be inadequate for the staging of MALT lymphoma, since it does not incorporate information on the depth of tumor invasion, known to affect prognosis. The Lugano staging system is the most widely accepted staging system, which incorporates measures of distant nodal involvement. Early disease (stage I-II) includes a single primary lesion or multiple, noncontiguous lesions confined to the GI tract that may have nodal involvement. There is no stage III. Advanced disease (stage IV) displays disseminated extranodal involvement or concomitant supra-diaphragmatic nodal involvement. ${ }^{23}$ Treatment of gastric MALT lymphoma is guided by the presence or absence of Hp infection. Several small prospective, single-arm trials and larger retrospective series have evaluated the use of antibiotics and PPIs for treatment of gastric MALT lymphoma. ${ }^{6,24-26}$ Almost all patients were able to successfully eradicate $\mathrm{Hp}$ infection, but $20 \%$ required a second course of Hp directed therapy. Histologic complete remission was achieved in $50-83 \%$ of patients. The median time from Hp infection to $\mathrm{CR}$ was 15 months, but it ranged from 5 months to 3 years. In our series, CR at 1 and 2 years was achieved at 6 and 8,5 months, respectively. Patients treated primarily with radiotherapy (RT) almost always attain a CR and have lower relapse rates $(<5 \%)$, but have a potential for more complications. ${ }^{27}$

After Hp eradication therapy, it is necessary to determine whether Hp was successfully eradicated (4 to 8 weeks after completion of therapy, with urea breath test or stool antigen). Patients must be monitored with periodic endoscopies with multiple biopsies to evaluate for tumor response and recurrence. ${ }^{7}$ The exact time interval and the duration of surveillance are not yet known but it is suggested to repeat endoscopy every 6 months for the first 2 years. ${ }^{28}$ Treatment failures $(20-30 \%)$ or relapses during follow-up should be treated with local RT with curative intent. ${ }^{27,28}$ The appropriate timing of second line therapy is difficult due to the sometimes slow response to $\mathrm{Hp}$ eradication therapy. If disease is still present after 12-18 months, patients should be referred to RT. Recently, it has been shown that the majority of patients with histological residuals of gastric MALT lymphoma after successful eradication, can be successfully managed by a watch-and-wait strategy with regular endoscopies with biopsies.
Patients with lymphomas demonstrating the $\mathrm{t}(11 ; 18)$ translocation are unlikely to respond to $\mathrm{Hp}$ eradication and are candidates for alternative therapies. ${ }^{29}$ A minority of patients have early stage (Lugano I/II) Hpnegative infection. These patients should be treated with local RT (25-30 Gy) with curative intent, which results in high rates of overall and complete response (100 and $98 \%$, respectively). ${ }^{27,28}$ For patients failing or recurring after RT, other treatments are reserved, including immunotherapy with rituximab (overall and complete response rates of 77 and $46 \%$, respectively) $)^{30,31}$ and chemotherapy with oral cyclophosphamide or chlorambucil (CR rates of $75 \%$ ) or intravenous cladribine (CR rate of $84 \%){ }^{32}$

According to literature, the majority of patients with gastric MALT lymphoma present with early stage (Lugano I-II) Hp-positive disease, differently from Hp-negative patients, which commonly present with advanced stages at diagnosis. ${ }^{3-7}$ In our series, only $59 \%$ had histological evidence of Hp infection and 91\% had limited disease according to Lugano staging system. In this group (Lugano I-II), Hp-positive patients achieved less CR at one year when compared to Hp-negative patients (67\% versus $92 \%)$. These results can express a geographic difference or the faster effect of more aggressive therapies in Hp-negative patients. In addition, the small number of patients, the short period of follow-up and the heterogeneous protocols used in each Center can also be implicated. As in our series, literature reports anecdotic cases of CR in Hp-negative MALT lymphoma treated only with antibiotics and PPIs (typically with limited disease). In a recent pooled analysis of data from 11 studies with 110 patients, eradication treatment achieved complete regression of lymphoma in $15,6 \%$ of Hp-negative patients. ${ }^{33}$ However, we should have in account that the most plausible explanation is that they are false negatives, or that this phenomenon is a consequence of therapy on microorganisms others than $\mathrm{Hp}$, which may have a similar proliferative effect on the lymphoma and may be responsive to antibiotics.

Treatment of patients with advanced disease (Lugano IV) is not clearly defined. ${ }^{7,28}$ As a result from different protocols used in our centers, a bias in our analysis could have occurred. These patients were treated with RT, rituximab or chemotherapy with or without RT. In this group, we report rates of CR of $66 \%$ in one year, occurring only in Hp-positive patients. Surgery is typically reserved for patients with complications such as perforation or obstruction; ${ }^{7}$ in our series, 2 patients $(6 \%)$ needed surgery due to perforation and bleeding that could not be controlled by endoscopic techniques. We evaluated several clinical and laboratorial factors that could predict CR in oneyear. Although we could not reach a statistic significance, probably due to the limited number of our population, a trend was observed for the variables younger age, low leukocyte count, IPI score $<3$ and limited disease. Several authors reported that the major negative predictive factor of complete regression rate was the presence of perigastric lymph nodes on EUS. ${ }^{34}$ Neoplasia stage, depth of infiltration in the gastric wall, presence of the API2-MALT1 translocation, and patient ethnicity has been identified as predictors of remission in a recent study. ${ }^{35}$ Our study reinforces MALT lymphomas as a notoriously indolent disease. To our knowledge, no previous report evaluated clinical and laboratorial factors for achieving complete remission in a Portuguese population with gastric MALT lymphoma.

\section{Conclusion}

Our results confirm the good prognosis and indolence of primary gastric MALT lymphoma, even in cases of advanced disease. Only $59 \%$ of the patients were Hp-positive. The most probable explanation 
is a missed diagnosis caused by a single test used for the diagnosis of Hp infection, its variable distribution in the stomach and the concomitant use of PPIs, which can lead to false-negative results. The factors studied could not predict CR in one-year, but it seems to exist a trend for achieving remission in younger patients, and in those with low leukocyte count, IPI score $<3$ and limited disease Patients with early stage Hp-negative MALT lymphoma and those with advanced disease, might still benefit from antibiotic treatment.

\section{Acknowledgments}

None.

\section{Conflicts of interest}

The author declares there is no conflict of interest.

\section{References}

1. Freeman C, Berg JW, Cutler SJ. Occurrence and prognosis of extranodal lymphomas. Cancer. 1972;29(1):252-260.

2. Swerdlow SH, Campo E, Harris NL, et al. WHO classification of tumours of haematopoetic and lymphoid tissues. (4th edn), IARC Press, USA, 2008. p. 439.

3. Wotherspoon A, Ortiz-Hidalgo C, Falzon MR, et al. Helicobacter pylori associated gastritis and primary B-cell lymphoma. Lancet. 1991;338(8776): 1175-1176.

4. Chan JK, Ng CS, Isaacson PG. Relationship between high-grade lymphoma and low-grade B-cell mucosa associated lymphoid tissue lymphoma of the stomach. Am J Pathol. 1990;136(5):1153-1164.

5. Eck M, Schmausser B, Haas R, et al. MALT-type lymphoma of the stomach is associated with Helicobacter pylori strains expressing the CagA protein. Gastroenterology. 1997;112(5):1482-1486.

6. Wotherspoon AC, Doglioni C, Diss TC, et al. Regression of primary low-grade B-cell gastric lymphoma of mucosa-associated lymphoid tissue. Lancet. 1993;342(8871):575-577.

7. Fischbach W. Best Practice \& Research Clinical Gastroenterology. 2014;28:1069-1077.

8. Malfertheiner P, Megraud F, O'Morain CA. Management of Helicobacter pylori infection -the Maastricht IV/ Florence Consensus Report. Gut. 2012;61:646.

9. Dawson I, Cornes J, Morson B. Primary malignant lymphoid tumours of the intestinal tract. Report of 37 cases with a study of factors influencing prognosis. Br J Surg. 1961;49:80-89.

10. Shiozawa E, Norose T, Kaneko K, et al. Clinicopathological comparison of the World Health Organization/Wotherspoon score to the Groupe d'Etude des Lymphomes de l'Adult grade for the post-treatment evaluation of gastric mucosa-associated lymphoid tissue lymphoma. $J$ Gastroenterol Hepatol. 2009;24(2):307-315.

11. Koch P1, del Valle F, Berdel WE, et al. Primary gastrointestinal nonHodgkin's lymphoma: I. Anatomic and histologic distribution, clinical features, and survival data of 371 patients registered in the German Multicenter Study GIT NHL 01/92. J Clin Oncol. 1991;19(18):38613873.

12. Cogliatti SB, Schmid U, Schumacher U, et al. Primary B-cell gastric lymphoma: a clinicopathological study of 145 patients. Gastroenterology. 1991;101(5):1159-1170.

13. Kolve M, Fischbach W, Greiner A, et al. Differences in endoscopic and clinicopathological features of primary and secondary gastric nonHodgkin's lymphoma. Gastrointest Endosc. 1999;49(3):307-315.
14. Muller AF, Maloney A, Jenkins D, et al. Primary gastric lymphoma in clinical practice. Gut. 1995;1973-1992. Gut 36(5): 679-683.

15. Wang T, Gui W, Shen Q. Primary gastrointestinal non-Hodgkin's lymphoma: clinicopathological and prognostic analysis. Med Oncol. 2010;27(3):661-683.

16. Radaszkiewicz Th, Dragosics B, Bauer P. Gastrointestinal malignant lymphomas of the mucosa-associated lymphoid tissue. Factors relevant to prognosis. Gastroenterology. 1992;102(5):1628-1638.

17. Stolte M. Helicobacter pylori gastritis and gastric MALT-lymphoma. Gut. 1992;50(S3):11119-11124.

18. Morgner A, Lehn N, Andersen LP, et al. Helicobacter heilmanniiassociated primary gastric low-grade MALT lymphoma: complete remission after curing the infection. Gastroenterology. 2000;118(5):821-828.

19. Zucca E, Bertoni F, Roggero E, et al. Molecular analysis of the progression from Helicobacter pylori-associated chronic gastritis to mucosa-associated lymphoid-tissue lymphoma of the stomach. $N$ Engl J Med. 1998;338(12) 804-810.

20. Akamatsu T, Mochizuki T, Okiyama Y, et al. Comparison of localized gastric mucosa-associated lymphoid tissue (MALT) lymphoma with and without Helicobacter pylori infection. Helicobacter. 2006;11(2):86-95.

21. Ruskon FA, Fischbach W, Aleman BMP, et al. EGILS consensus report. Gastric extranodal marginal zone B cell lymphoma of MALT. Gut. 2011;60(6):747-758.

22. Fischbach W, Al-Taie O. Staging role of EUS. Best Pract Res Clin Gastroenterol. 2010;24(1):13-17.

23. Rohatiner A, d'Amore F, Coiffier B, et al. Report on a workshop convened to discuss the pathological and staging classifications of gastrointestinal tract lymphoma. Ann Oncol. 1994;5(5):397-400.

24. Wündisch T, Thiede C, Morgner A, et al. Long-term follow-up of gastric MALT lymphoma after Helicobacter pylori eradication. $J$ Clin Oncol. 2005;23(31):8018-8024.

25. Stathis A, Chini C, Bertoni F, et al. Long-term outcome following Helicobacter pylori eradication in a retrospective study of 105 patients with localized gastric marginal zone B-cell lymphoma of MALT type. Ann Oncol. 2009;20(6):1086-1093.

26. Nakamura S, Sugiyama T, Matsumoto T, et al. Long-term clinical outcome of gastric MALT lymphoma after eradication of Helicobacter pylori: a multicentre cohort follow-up study of 420 patients in Japan. Gut. 2012;61(4):507-513.

27. Tomita N, Kodaira T, Tachibana H, et al. Favorable outcomes of radiotherapy for early-stage mucosa-associated lymphoid tissue lymphoma. Radiother Oncol. 2009;90(2):231-235.

28. Zucca E, Copie-Bergman C, Ricardi U, et al. Gastric marginal zone lymphoma of MALT type: ESMO clinical recommendations for diagnosis, treatment and follow-up. Ann Oncol Suppl. 2008;6:144-148.

29. Liu H, Ye H, Ruskone-Fourmestraux A, et al. $\mathrm{T}(11 ; 18)$ is a marker for all stage gastric MALTlymphomas that will not respond to H. pylori eradication. Gastroenterology. 2002;122(5):1286-1294.

30. Martinelli G, Laszlo D, Ferreri AJ, et al. Clinical activity of rituximab in gastric marginal zone non-Hodgkin's lymphoma resistant to or not eligible for anti-Helicobacter pylori therapy. J Clin Oncol. 2005;23(9):1979-1983.

31. Conconi A, Martinelli G, Thiéblemont C, et al. Clinical activity of rituximab in extranodal marginal zone B-cell lymphoma of MALT type. Blood. 2003;102(8):2741-2745. 
32. Olszewski AJ, Castillo JJ. Comparative outcomes of oncologic therapy in gastric extranodal marginal zone (MALT) lymphoma: analysis of the SEER-Medicare database. Ann Oncol. 2013;24(5):1352-1359.

33. Zullo A, Hassan C, Ridola L, et al. Eradication therapy in Helicobacter pylori-negative,gastric low-grade mucosa-associated lymphoid tissue lymphoma patients. A systematic review. J Clin Gastroenterol. 2013;47(10):824-827.
34. Levy M, Copie-Bergman C, Traulle C, et al. Groupe d'Etude des Lymphomes de l'Adulte (GELA). Conservative treatment of primary gastric low-grade B-cell lymphoma of mucosa-associated lymphoid tissue: predictive factors of response and outcome. Am J Gastroenterol. 2002;97(2):292-297.

35. Angelo Zullo, Cesare Hassan, Lorenzo Ridola, et al. Gastric MALT lymphoma: old and new insights. Ann Gastroenterol. 2014;27(1):2733 